\title{
Valoración socio-ambiental y propuesta de gestión de una alternativa técnica y ambientalmente viable para la mitigación de contaminantes en el río Tinajillas del cantón Rumiñahui, Pichincha-Ecuador
}

\author{
CHRISTIAN PAREDES-ILLANESa* , MARCO ANTONIO ZALDUMBIDE VERDEZOTO ${ }^{a}$ \\ a Universidad Central del Ecuador, Facultad de Ingeniería en Geología, Minas Petróleos y Ambiental. \\ Ciudadela Universitaria, Calle Jerónimo Leyton y Av. La Gasca Quito / Ecuador. \\ *Correspondencia: chrisxavquimicoecuador@gmail.com
}

Recibido: 21 marzo 2016, Aceptado: 17 agosto de 2016

\section{Resumen}

Las aguas del río Tinajillas, en el cantón Rumiñahui (Pichincha-Ecuador) están siendo contaminadas con descargas líquidas y sólidas de origen doméstico, mecánicas automotrices y faenamiento clandestino de animales sin ningún tratamiento, convirtiéndose esto en un problema socio-ambiental. Ante ello, y con el fin de realizar una propuesta de gestión de una alternativa técnica y ambientalmente viable para controlar y mitigar la contaminación del agua de este río se determinó la calidad de sus aguas mediante métodos físico-químicos, microbiológicos y bióticos (macroinvertebrados) en 6 y 3 puntos, respectivamente. Los resultados indican que el agua de este río está contaminada por descargas de origen doméstico por lo que se valoraron dos propuestas de gestión para su tratamiento: sistema de tratamiento de aguas residuales aerobio y por fitorremediación con humedales. La segunda propuesta fue la más idónea por su bajo costo de mantenimiento y la creación de nuevos biotipos, su construcción se llevaría a cabo en el río aplicando técnicas de ingeniería naturalística.

Palabras clave: Tinajillas, aguas residuales domésticas, macroinvertebrados, fitorremediación, humedales.

\section{Socio-environmental assessment and management proposal of a technical alternative and environmentally viable for mitigating of pollutants in the river Tinajillas of Rumiñahui canton, Pichincha-Ecuador}

\begin{abstract}
The River Tinajillas in the Rumiñahui canton (Pichincha-Ecuador) is constantly being polluted with liquid and solid domestic discharges from private households, car repair shops and clandestine slaughter houses. Most of these discharges enter the river directly without any previous treatment causing considerable damage to the river's fauna and flora, affecting both natural scenery and quality of life of the residents who live next to the river and beyond. In an attempt to measure the environmental impact of the pollution, six water samples were analyzed in laboratory using physic-chemical and microbiological methods, besides 3 water samples containing biological components were examined in order to evaluate the quality of Tinajillas's waters. The laboratory results of analysis show a highly polluted water which does not meet the standards set in the Ecuadorian Environmental Legislation for Water. To recover the water quality of this river, two management proposals are presented. First, an aerobic treatment plant and second, a phytoremediation wetlands treatment. The second one turned out to be the best option because of its low cost and easy maintenance, besides it enhances the creation of new biotopes and does not generate any odor, and could be built using bioengineering techniques.
\end{abstract}

Keywords: Tinajillas, domestic wastewaters, macroinvertebrate, phytoremediation, wetlands 


\section{Introducción}

El río Tinajillas, ubicado en la provincia de Pichincha, en Ecuador, tiene una longitud de $5 \mathrm{~km}$. Nace en el barrio Albornoz al sur de Sangolquí y desemboca en el río San Nicolás, la unión de estos dos toma el nombre de río Cachaco que finalmente se une al río San Pedro. El río Tinajillas está siendo contaminado por la generación de descargas líquidas y sólidas de origen doméstico de la población sin ningún tratamiento previo, convirtiéndose en un foco de problemas de salud pública por la presencia de vectores (roedores, moscos) y mal olor.

Este río carece de estudios ambientales relativos al comportamiento hidrológico, calidad de sus aguas, estado de sus riberas, caudal promedio anual, existencia de flora y fauna y el aspecto socio-ambiental. Con esta investigación se pudo conocer la situación actual de la calidad de sus aguas mediante análisis físico-químicos, microbiológicos y bióticos (macroinvertebrados) tanto en campo como en el laboratorio, los resultados ayudaron en el planteamiento de la propuesta de gestión con alternativas técnicas y ambientalmente viables tales como sistema de tratamiento [1] por lagunas de oxidación y fitorremediación con humedales de flujo superficial [2] para controlar y mitigar el grado de contaminación de sus aguas y mejorar el paisajismo mediante una metodología de tratamiento adecuada y con esto dar solución a un problema socio-ambiental existente.

El sistema de tratamiento de aguas residuales domésticas por lagunas de oxidación más conocido en el Ecuador es la planta de tratamiento UCUBAMBA en la ciudad de Cuenca, trata el $95 \%$ de aguas residuales de dicha ciudad [3], es un sistema bueno pero costoso por lo que es necesario considerar métodos que sean ambientalmente amigables y de bajo costo como la fitorremediación con humedales que resulta ser eficaz en la remoción de materia orgánica, elementos patógenos y nutrientes [4] cuya construcción hace uso de técnicas basadas en la ingeniería naturalística [5], este sistema de tratamiento es aplicado en varios países de Europa y está siendo construido en la comunidad de Chazojuan, provincia de Bolívar.

\section{Materiales y métodos}

\section{Toma de muestras}

Para la toma de muestras tanto de agua como del componente biótico se estableció el criterio de mayor influencia de descargas a lo largo del río Tinajillas identificando de esta manera 6 puntos de muestreo de agua y 3 puntos de muestreo de macroinvertebrados y peces (Figura 1), dichos puntos fueron identificados satelitalmente con el sistema de posicionamiento global (GPS, por sus siglas en inglés) en coordenadas geográficas UTM WGS84 (Tabla 1).
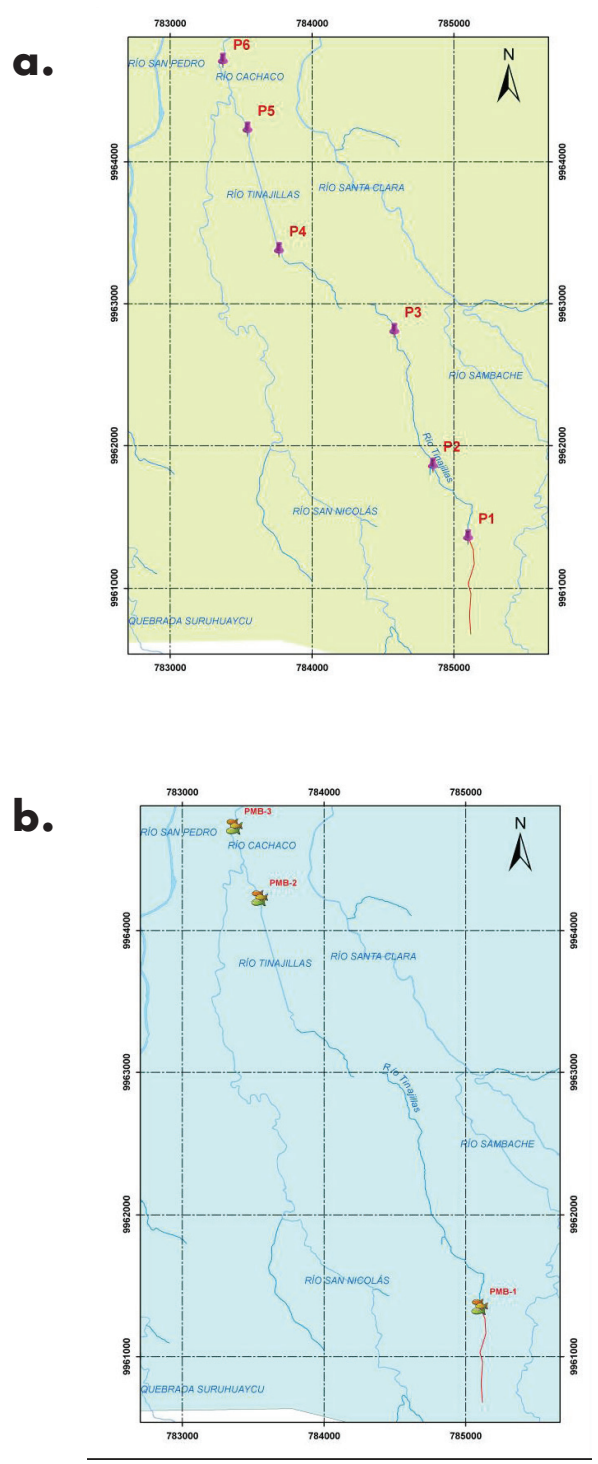

Figura 1. a. Ubicación geográfica de los puntos de muestreo de agua y $\mathbf{b}$. Ubicación geográfica del componente biótico del río Tinajillas 
Teniendo en cuenta las medidas de bioseguridad, se tomaron en primer lugar las muestras para el análisis microbiológico en las fundas estériles específicas para este fin, luego se tomaron las muestras para el análisis físico-químico introduciendo los recipientes de plástico y vidrio ámbar nuevos en el centro del río hasta llenarlos y luego de etiquetarlos se transportaron en un contenedor a $4^{\circ} \mathrm{C}$ para conservar las condiciones originales de las muestras. Los datos tomados en campo fueron la temperatura, $\mathrm{pH}$ y conductividad con un potenciómetro y el caudal (Tabla 1) se determinó aplicando el método de velocidad de corriente y el área de sección transversal. Las 3 muestras de macroinvertebrados fueron tomadas con una red Surber colocando la misma en el fondo del río en sentido contrario a la corriente y removiendo dinámicamente el sustrato por delante con los pies en diferentes partes del punto de muestreo para luego colocar el sustrato en frascos etiquetados y con alcohol al $75 \%$ (Figura 2). No se tomaron muestras de peces debido al alto grado de contaminación del río.
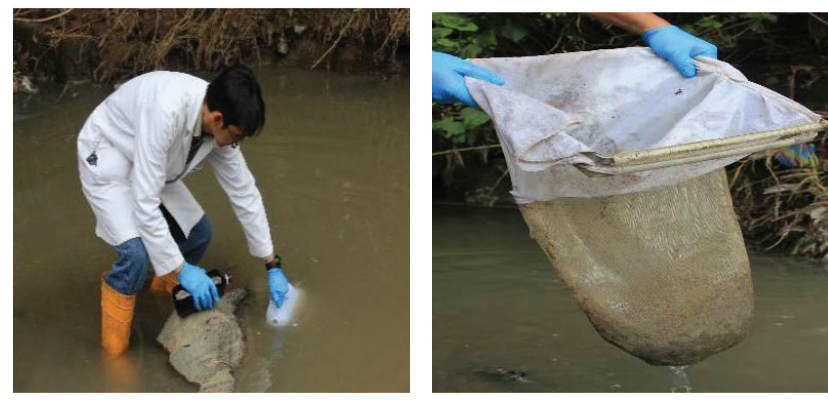

Figura 2. De izquierda a derecha: Toma de muestras de agua y de muestras de macroinvertebrados en el río Tinajillas.

\section{Análisis de muestras}

Los análisis se realizaron en un laboratorio público acreditado. Para los análisis de las muestras de agua se siguieron los métodos establecidos en el laboratorio tales como Absorción Atómica, Espectroscopia
Infrarroja, Espectroscopia UV-Visible, Titulométrico y Extracción con solventes orgánicos, donde se hizo uso de material volumétrico de vidrio, reactivos como cloroformo, tiosulfato de sodio, ácido nítrico concentrado, ácido clorhídrico diluido, disolvente S316, hexano y equipos específicos como espectrofotómetro de absorción atómica, equipo de filtración al vacío, espectrofotómetro $\mathbb{R}$, digestor para $D Q O$, fotómetro UV visible y autoclave. En el laboratorio se analizaron 19 parámetros físico-químicos y 2 microbiológicos en las 6 muestras de agua recolectadas tales como: Demanda Química y Bioquímica de Oxígeno, arsénico, cadmio, mercurio, níquel, cromo total, cromo $\mathrm{Vl}$, plomo, hidrocarburos totales de petróleo, tensoactivos, nitritos, nitratos, nitrógeno total, oxígeno disuelto, sólidos suspendidos, sólidos totales, sólidos disueltos y coliformes totales y fecales.

Dentro de esta investigación también se realizó el reconocimiento de la flora y fauna existente a lo largo de este río para lo cual se fotografió tanto plantas como aves y mamíferos encontrados y posteriormente se comparó con bibliografía [6] de flora y fauna para su identificación taxonómica.

La investigación puso énfasis en el aspecto ambiental y social por lo que también se realizó una encuesta socio ambiental a 132 moradores del sector quienes están afectados directamente, $y$, finalmente, se realizó una evaluación del impacto ambiental [7] de la zona de estudio basado en la técnica de Leopold para conocer los efectos potenciales del proyecto de tratamiento de aguas residuales domésticas que se llevaría a cabo. La flora, fauna, calidad del agua y aspecto social son componentes que ayudaron en la determinación de la línea base (descripción de la situación actual sin nuevas intervenciones antrópicas); información que ayudó en la propuesta de gestión de una alternativa técnica y ambientalmente viable para la recuperación y mitigación de la contaminación del agua del río Tinajillas del cantón Rumiñahui.

Tabla 1. Datos geográficos y caudales de los puntos de muestreo.

\begin{tabular}{ccccccc}
\hline & Punto 1 & Punto 2 & Punto 3 & Punto 4 & Punto 5 & Punto 6 \\
\hline Altitud, msnm & 2561 & 2539 & 2525 & 2510 & 2492 & 2481 \\
Coordenadas (WGS 84) & 785098 & 784848 & 784580 & 783768 & 783545 & 783372 \\
Caudal, m ${ }^{3} / \mathrm{s}$ & 9961352 & 9961862 & 9962809 & 9963380 & 9964229 & 9964714 \\
& 0,012 & 0,034 & 0,091 & 0,39 & 0,402 & 2,16 \\
\hline
\end{tabular}


Los resultados físico-químicos y microbiológicos (Figuras 3,4 y 5) se compararon con los límites máximos permisibles establecidos en la Norma ambiental ecuatoriana, libro VI Anexo I del Texto Unificado de Legislación Secundaria de Medio Ambiente (TULSMA): Criterios de calidad admisibles para la preservación de la flora y fauna en aguas dulces y Criterios de calidad admisibles para aguas de uso agrícola.

\section{Resultados y discusión}

\section{Aspecto químico}

En la Tabla 2 se resumen los resultados del análisis físico-químico de los seis puntos de muestreo del río
Tinajillas. Estos indican la presencia de contaminantes tanto de origen orgánico como inorgánico que afectan a la calidad del agua del río, principalmente a la fauna acuática por la presencia de materia orgánica, aceites y grasas, tensoactivos e hidrocarburos totales de petróleo. El oxígeno disuelto es casi nulo lo cual impide el desarrollo de peces, hay invasión de otros organismos como los macroinvertebrados que se adaptan a condiciones alteradas del agua.

Tabla 2. Resultados del análisis físico-químico de los seis puntos de muestreo de agua del río Tinajillas.

\begin{tabular}{|l|c|c|c|c|c|c|c|}
\hline \multicolumn{1}{|c|}{ PARÁMETROS } & Punto 1 & Punto 2 & Punto 3 & Punto 4 & Punto 5 & Punto 6 & $\begin{array}{c}\text { LíMITE } \\
\text { PERMISIBLE }\end{array}$ \\
\hline Arsénico(mg/L) & 0,000775 & 0,00189 & 0,001434 & 0,001588 & 0,001712 & 0,00949 & 0,05 \\
\hline Cadmio (mg/L) & $<0,02$ & $<0,02$ & $<0,02$ & $<0,02$ & $<0,02$ & $<0,02$ & 0,001 \\
\hline Color & 154 & 131 & 103 & 84 & 123 & 79 & $\ldots$. \\
\hline Conductividad (uS/cm) & 508 & 593 & 589 & 525 & 526 & 471 & $\ldots$. \\
\hline Cromo Total (mg/L) & $<0,04$ & $<0,04$ & $<0,04$ & $<0,04$ & $<0,04$ & $<0,04$ & 0,05 \\
\hline Cromo VI (mg/L) & $<0,025$ & $<0,025$ & $<0,025$ & $<0,025$ & $<0,025$ & $<0,025$ & $\ldots$. \\
\hline DQO (mg/L) & 321 & 230 & 174 & 135 & 190 & 128 & 100 \\
\hline DBO (mg/L) & 128 & 138,08 & 91,8 & 67,34 & 82,81 & 54,49 & 250 \\
\hline Aceites y Grasas (mg/L) & 16,2 & 8 & 4,4 & 2,2 & 11,2 & 5,6 & 0,3 \\
\hline TPH (mg/L) & 1,9 & 1,5 & 0,5 & $<0,5$ & 1,3 & $<0,5$ & 0,5 \\
\hline Mercurio (mg/L) & 0,0012 & 0,0004 & 0,0032 & 0,0006 & 0,0004 & 0,0008 & 0,0002 \\
\hline Nitritos (mg/L) & $<0,010$ & $<0,010$ & $<0,010$ & $<0,010$ & $<0,010$ & 0,02 & $\ldots$. \\
\hline Nitratos (mg/L) & 13,6 & 5,9 & 7,2 & 7 & 9 & 4,6 & 10 \\
\hline Nitrógeno Total (mg/L) & 14,1 & 19,2 & 25,0 & 18,4 & 17,2 & 11,7 & 15 \\
\hline Níquel (mg/L) & $<0,16$ & $<0,16$ & $<0,16$ & $<0,16$ & $<0,16$ & $<0,16$ & 0,025 \\
\hline Oxígeno Disuelto (mg/L) & 0,350 & 1,09 & 1,1 & 1,24 & 1,83 & 0,46 & $>6 \mathrm{mg} / \mathrm{L}$ \\
\hline pH & 8,2 & 8,3 & 9,6 & 9,6 & 9,7 & 8,28 & $6,5-9$ \\
\hline Plomo (mg/L) & $<0,09$ & $<0,09$ & $<0,09$ & $<0,09$ & $<0,09$ & $<0,09$ & 0,2 \\
\hline Sólidos Suspendidos (mg/L) & 155 & 82,5 & 75 & 51,66 & 155 & 67 & 100 \\
\hline Sólidos Disueltos (mg/L) & 293 & 329 & 302 & 262 & 283 & 36 & 3000 \\
\hline Sólidos Totales (mg/L) & 345 & 442 & 406 & 348 & 422 & 475 & 1600 \\
\hline Tensoactivos (mg/L) & 3,285 & 4,488 & 2,3755 & 2,0055 & 2,1495 & 1,587 & 0,5 \\
\hline Temperatura (C) & 18,6 & 18,6 & 18,6 & 18,2 & 18,0 & 18,1 & 20 \\
\hline
\end{tabular}


La Demanda Bioquímica de Oxígeno $\left(\mathrm{DBO}_{5}\right)$ y Demanda Química de Oxígeno (DQO) son parámetros que determinan el grado de contaminación del agua de descarga ya sea de origen doméstico o industrial y su relación es un método que permite identificar el nivel de contaminación de las aguas según su origen, siendo $<0,2$ vertidos de origen industrial y $>0,5$ vertidos de origen urbano [8], en este estudio los resultados para los puntos 1,5 y 6 dieron un valor de 0,4 y para los puntos 2,3 y 4 dieron un valor de 0,6 y 0,5 , respectivamente, lo cual indica que los vertidos son mayoritariamente de origen urbano.
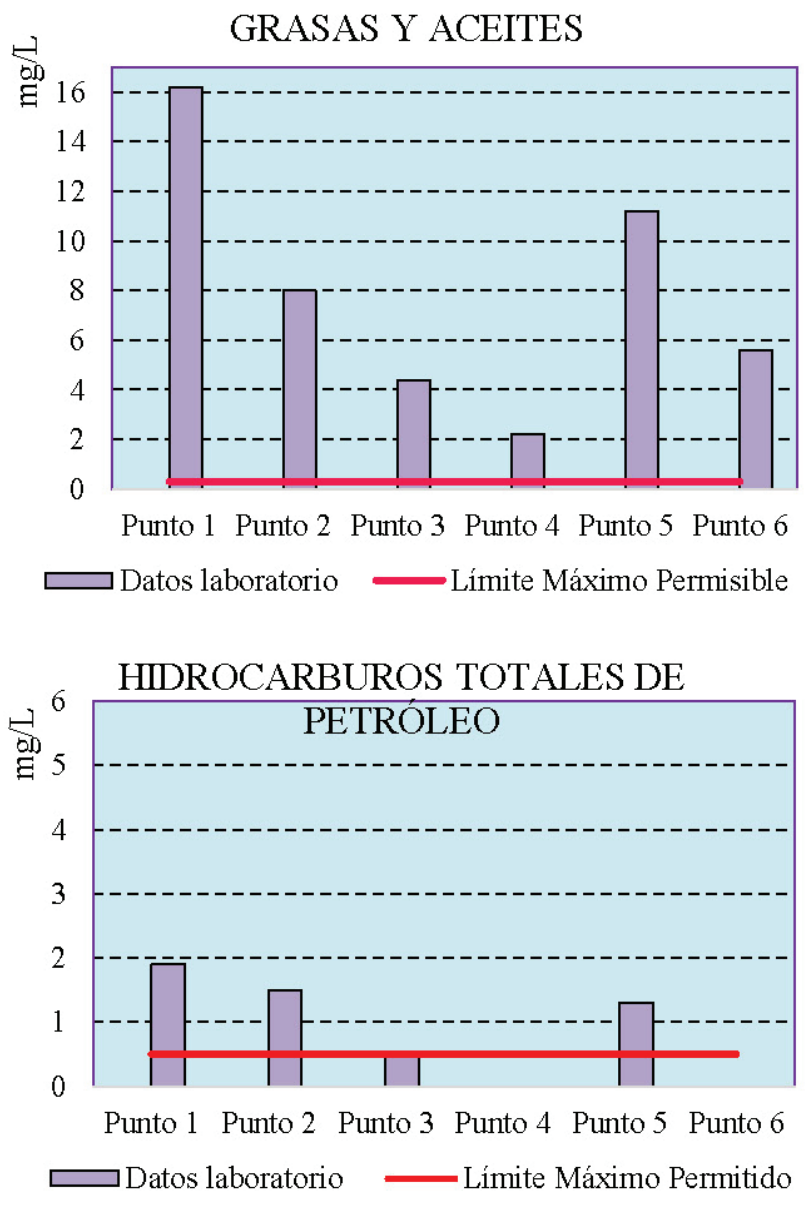

Figura 3. Resultados de aceites-grasas e hidrocarburos totales de petróleo de los seis puntos de muestreo de agua del río Tinajillas.

Los aceites y grasas e hidrocarburos totales de petróleo (TPH) (Figura 3) están presentes como residuos en las muestras de agua tomadas debido a las descargas domésticas y de mecánicas automotrices existentes en la zona de estudio, sus valores sobrepasan los límites máximos permisibles afectando directamente a la calidad del agua de este río debido a que estos contaminantes interfieren en la actividad biológica.
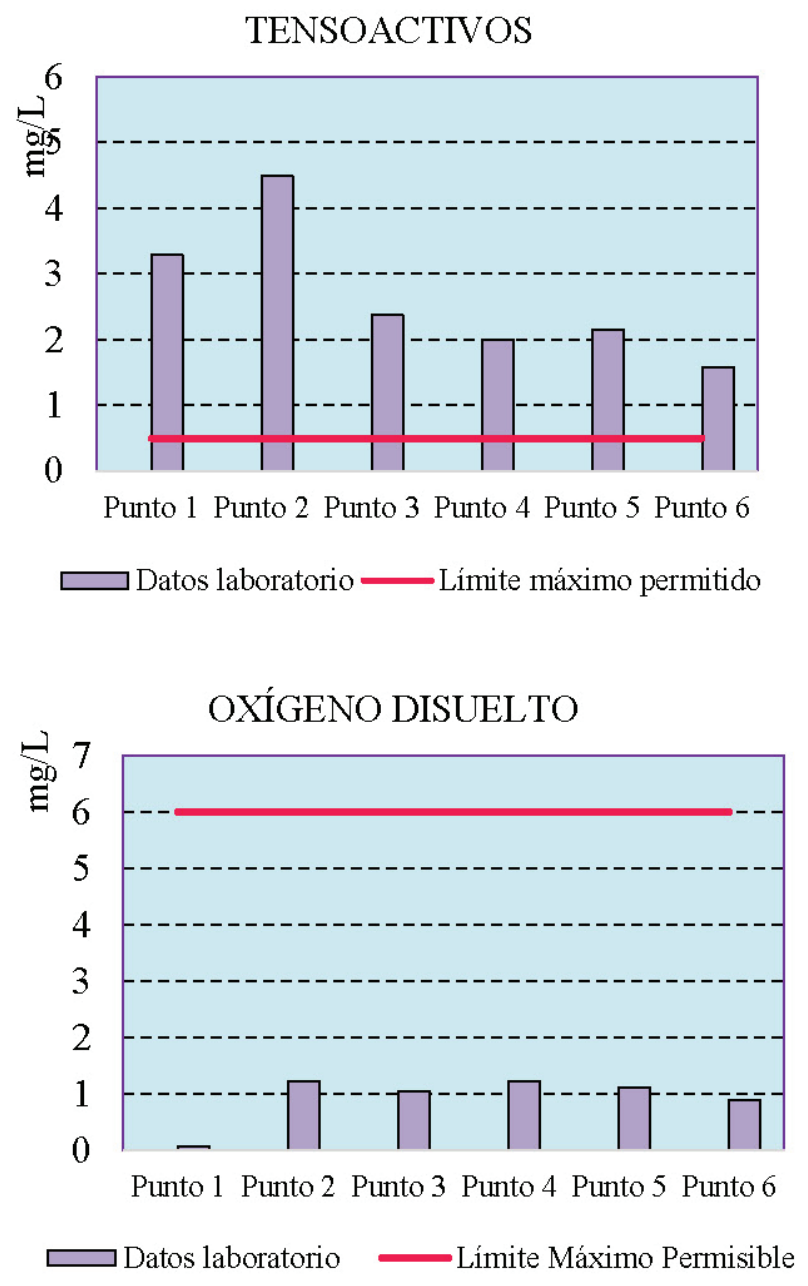

Figura 4. Resultados de tensoactivos y oxígeno disuelto de los seis puntos de muestreo de agua del río Tinajillas.

La presencia de residuos de detergentes y jabones (tensoactivos) sobrepasan los límites permisibles debido a las descargas de origen doméstico y de lavadoras de autos existentes en la zona de estudio ya que no tienen un sistema de tratamiento previo a la descarga al río. La concentración de oxígeno disuelto es casi nula, es decir, sus valores no llegan al límite mínimo requerido (6 $\mathrm{mgO}_{2} / L$ ) para el desarrollo de la vida acuática, situación debida principalmente a la presencia de materia orgánica producto de las descargas de origen doméstico, mecánicas automotrices y mataderos clandestinos (Figura 4). 


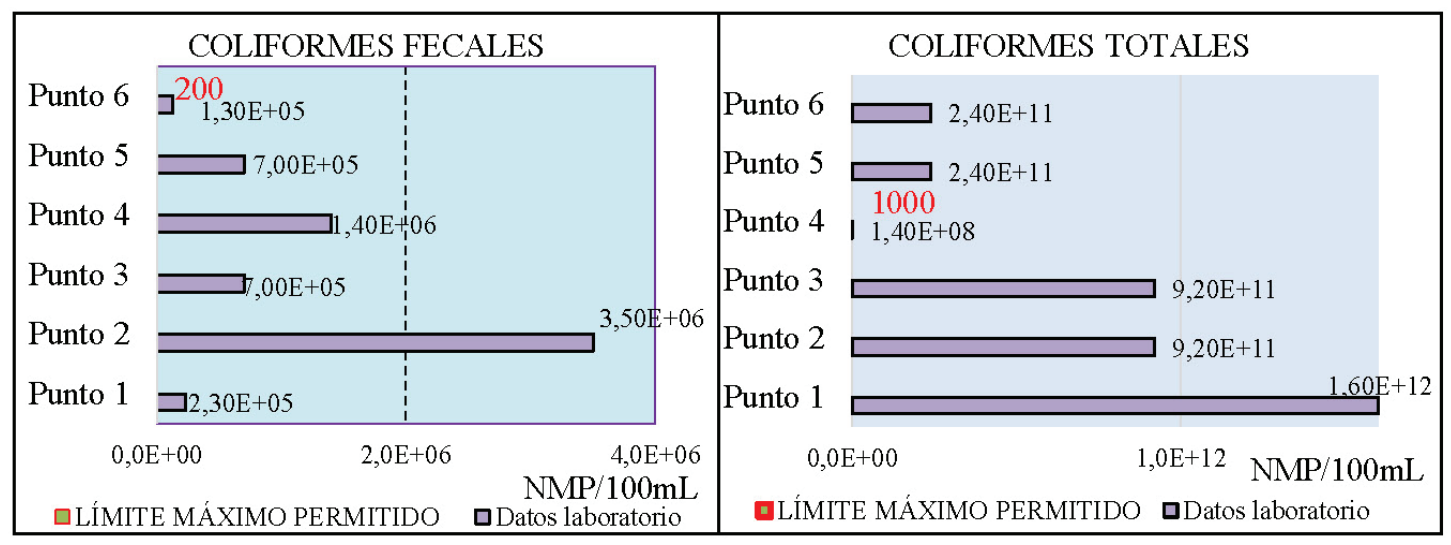

Figura 5. Resultados de análisis microbiológicos de laboratorio de los seis puntos de muestreo de agua del río Tinajillas.

Los resultados del análisis microbiológico (Figura 5) indican la presencia de microorganismos patógenos principalmente de origen fecal producto de las descargas de aguas negras y grises de las viviendas cercanas al río, convirtiendo la calidad del agua en no apta para ningún uso debido a que estos microorganismos patógenos son causantes de enfermedades.

La mayoría de moradores cercanos a la zona de estudio tienen un sistema de alcantarillado combinado, es decir, las descargas líquidas domésticas y aguas lluvia van directamente al río aumentando la carga contaminante e impidiendo su auto depuración provocando que la cantidad de oxígeno disuelto disminuya y sea la causa para que exista mal olor debido a la descomposición de la materia orgánica. A las pruebas anteriores para determinar la calidad del agua se suma el componente biótico con los macroinvertebrados, bio-indicadores de mucha importancia en los cuerpos hídricos. En este estudio se encontraron 151 individuos agrupados en 2 órdenes, 3 familias y 4 morfoespecies, tales como Tanypodinae, Orthocladiinae, Tipula y Tubifex típicos de hábitats fuertemente contaminados o alterados. Situación que se evitaría al contar con un sistema de alcantarillado separado y un sistema de tratamiento de aguas domésticas ambientalmente viable como la fitorremediación.

\section{Aspecto biótico}

Se encontraron 5 especies de mamíferos: ratones, perros, gatos, caballos y vacas. Por otro lado, se evidenció la presencia de 10 especies de aves $[9,10]$ siendo las más notorias el Colibrí coruscans, Pyrocephalus rubinus (pájaro brujo), Sayornis nigricans (Febe guardarríos), Zenaida auriculata (tórtola), Zonotrichia capensis (gorrión), y Turdus fuscater (mirlo) (Figura 6). La presencia de aves y mamíferos está relacionada con la cantidad de área verde existente en la naturaleza, situación que en este caso es reducida ya que a lo largo del río los espacios verdes están ocupados por estructuras de cemento, principalmente viviendas. A pesar de esta situación, en las áreas verdes existentes a lo largo del río se encontraron 36 especies de plantas $[11,12]$ agrupadas en 18 familias, siendo las más comunes la Sida rhombifolia L. (escoba), Baccharis latifolia (chilca), Tanacetum parthenium (Santa María), Tilia glabra (tilo), Silybum marianum (cardo), Leonotis leonurus (cola de león) entre otras.
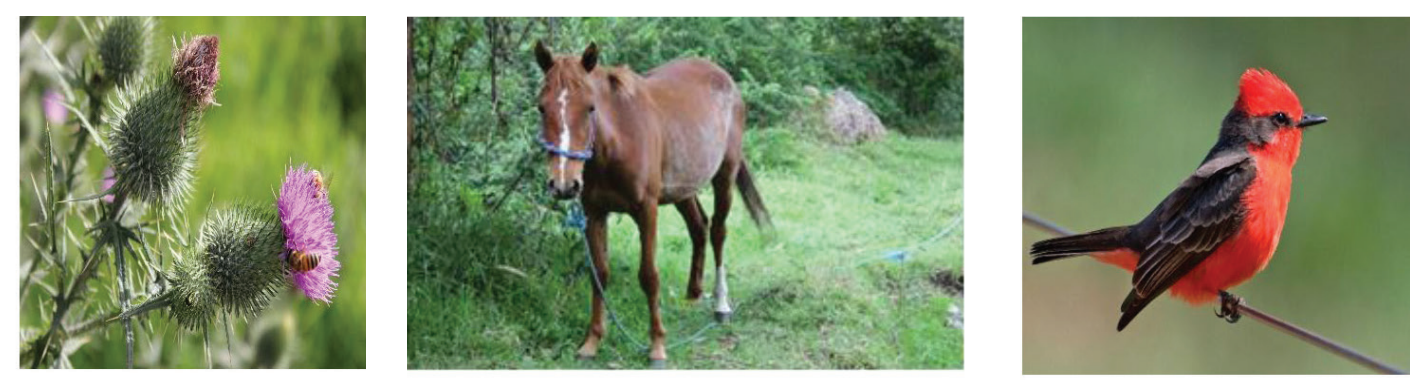

Figura 6. Biota encontrada en el sector, de izquierda a derecha: Silybum marianum (cardo), Equus ferus caballus (caballo) y Pyrocephalus rubinus (pájaro brujo). 


\section{Aspecto socio ambiental}

Las encuestas realizadas a los moradores de afectación directa se enfocaron en el aspecto socio ambiental, tomando una población de 200 y según el cálculo estadístico y con un nivel de significancia del $95 \%$ (1.96) y un error del 5\%, se tomó una muestra de 132 personas en la cual el $64 \%$ de los encuestados está de acuerdo en que los ríos, esteros y quebradas están siendo deteriorados por el impacto del hombre, así también el $64 \%$ de los encuestados está de acuerdo que se embaule el río debido principalmente a la presencia de malos olores, basura y roedores, lo que significa que habría que eliminar los espacios verdes, sin embargo el $58 \%$ de los encuestados está dispuesto a pagar para cambiar su sistema de alcantarillado combinado a uno separado con el fin de implementar una planta de tratamiento de aguas residuales domésticas y recuperar las aguas del río Tinajillas y, finalmente, el $83 \%$ de los encuestados pide que se conserven los espacios verdes y no se talen los árboles, lo cual significa que la mayoría de los moradores aprecian el medio ambiente.

\section{Alternativas de tratamiento de aguas residuales domésticas}

En la presente investigación se planteó dos alternativas de tratamiento de aguas residuales domésticas (Tabla 3), la alternativa 1 es el sistema de tratamiento aerobio con lagunas de oxidación, buen sistema pero con alto costo y con impactos negativos para el ambiente durante su construcción, y la alternativa 2 es el sistema de fitorremediación con humedales cuyo principio fundamental de purificación es el tratamiento biológico, en el cual las plantas son utilizadas como filtros naturales para la depuración de las aguas contaminadas, a la vez se recrea artificialmente el ambiente natural de los estanques de aguas.

Tabla 3. Cuadro comparativo de las características de las dos alternativas planteadas para la implantación del sistema de tratamiento de aguas residuales domésticas del río Tinajillas

\begin{tabular}{|c|c|c|c|}
\hline Factor & Características & Alternativa No. 1 & Alternativa No. 2 \\
\hline \multirow{7}{*}{ Técnico } & Área de terreno requerido, $\mathrm{m}^{2}$ & 20000 & 6000 \\
\hline & Cantidad de piscinas & 3 & 1 Humedal \\
\hline & Complejidad en la construcción & Alta & Mínima \\
\hline & Material de construcción requerido & Alto & Mínimo \\
\hline & Entrenamiento de personal & Medio & Mínima \\
\hline & Mano de obra calificada & Medio & Mínimo \\
\hline & Accesibilidad & Fácil & Fácil \\
\hline \multirow{7}{*}{ Económico } & $\begin{array}{l}\text { Compra de terreno para la construcción de la PTAR, } \\
\text { USD }\end{array}$ & 1928156.49 & $\begin{array}{l}\text { Nulo, su construcción sería } \\
\text { en el río }\end{array}$ \\
\hline & Estudios de ingeniería civil e hidráulica, USD & Alto & Medio \\
\hline & $\begin{array}{l}\text { Costos de material y mano de obra para la } \\
\text { construcción, USD }\end{array}$ & Alto & Medio \\
\hline & $\begin{array}{l}\text { Mantenimiento de la planta de tratamiento en } \\
\text { operación, USD }\end{array}$ & Alto & Mínimo \\
\hline & $\begin{array}{l}\text { Pago de sueldos al personal de planta y de apoyo, } \\
\text { USD }\end{array}$ & Alto & Medio \\
\hline & Financiamiento del proyecto & Alto & Medio \\
\hline & Consumo de energía eléctrica & Alto & Mínimo \\
\hline \multirow{3}{*}{ Ambiental } & Impactos ambientales en su construcción & Altos & Mínimos \\
\hline & Eliminación de malos olores & Alto & Bajo \\
\hline & Pasivos ambientales & Medio & Mínimo \\
\hline Socio económico & Generación de mano de obra & Medio & Medio \\
\hline \multirow{2}{*}{ Social } & Tratamiento de aguas contaminadas & Alto & Alto \\
\hline & Uso de suelos & Alto & Mínimo \\
\hline Paisajismo & Impacto visual & Alto & Mínimo \\
\hline Riesgos laborales & Accidentes durante la construcción y operación & Medio & Bajo \\
\hline
\end{tabular}


Las desventajas de implantar un sistema de tratamiento de aguas residuales por lagunas de oxidación para este río serían el elevado costo tanto por la adquisición del terreno como por la infraestructura, y la generación de olores indeseables para los pobladores cercanos ya que este sistema es a cielo abierto, mientras que el sistema por fitorremediación no genera malos olores debido a que el agua a ser tratada pasa por debajo de las plantas donde las raíces de las mismas actúan degradando la materia orgánica y su costo de mantenimiento es bajo.

\section{Conclusiones}

- Los resultados físico-químicos, microbiológicos y componente biótico indican que el agua del río Tinajillas está contaminada principalmente con materia orgánica, tensoactivos, hidrocarburos totales de petróleo, aceites y grasas y microorganismos patógenos de origen fecal y está alterada biológicamente lo cual define la ausencia de peces y que dicha agua no es apta para ningún uso. Al ser los vertidos mayoritariamente de origen urbano es posible aplicar tratamientos biológicos, por ejemplo con plantas fitodepuradoras.

- A lo largo del río no hay espacios verdes suficientes para el desarrollo de la fauna y flora por lo que su presencia es mínima: 5 especies de mamíferos, 10 especies de aves y 36 especies de plantas. Debido a esto los moradores encuestados están de acuerdo a que se implemente un sistema de tratamiento de aguas residuales sin quitar los espacios verdes existentes.

- La alternativa 2, remediación de aguas residuales por fitorremediación con humedales, es la más idónea para la implantación debido a que optimiza el espacio y recursos económicos, es de fácil mantenimiento, es amigable con el ambiente por lo que esto ayudaría a mejorar el paisajismo, crear nuevos biotipos y mantener el ecosistema del sector en equilibrio.

\section{Bibliografía}

[1]. Rojas Romero, J. A. (2010). Tratamiento de aguas residuales Teoría y principios de diseño. Colombia: Escuela Colombiana de Ingeniería.

[2]. ARTEC. (Febrero de 2016). Il flusso superficiale orizzontale. Obtenido de Gestione Sostenibile de la Risorsa Acqua: www.artecambiente.it/il-flusso-superficiale-orizzontale

[3]. ETAPA. (Diciembre de 2015). Planta de Tratamiento de Aguas Residuales. Obtenido de Empresa Pública Municipal de Telecomunicaciones, Agua Potable, Alcantarillado y Saneamiento: http://www.etapa.net.ec/

[4]. Davide Tocchetto, P. (2010). Developing Wastewater Reuse Practices. Wastewater Management.

[5]. Paesaggio e architettura rurale. (Febrero de 2016). Ingeniería Naturalística. Obtenido de Progretti Realizzati: www.paesaggio.net.

[6]. Rumiñahui, G. A. (2011). Atlas de Biodiversidad Cantón Rumiñahui. 146.

[7]. Larry W. Canter, P. (2010). Manual de Evaluación de Impacto Ambiental. Oklahoma: McGraw Hill.

[8]. Kenbo (Febrero de 2016). Niveles de contaminación de las aguas residuales por relación de $\mathrm{DBO}_{5}$ y DQO. Obtenido de Ineman S.A: http://kenbi.eu/kenbipedia_3.php?seccion=kenbipedia\&capitulo=3\#

[9]. Argentavis. (Febrero de 2016). Colibri coruscans. Obtenido de Argentavis - Aves de Argentina : http://argentavis.org/2012/sitio/masinfo.php?id=14

[10]. Argentavis. (Enero de 2016). Pyrocephalus rubinus (pájaro brujo). Obtenido de Argentavis Aves de Argentina: http://argentavis. org/2012/sitio/especie_dg.php?id=385

[11]. Plantas Medicinales. (Febrero 2016). Tilia glabra (Tilo). Obtenido de: http://www.tusplantasmedicinales.com/tilo/

[12]. The Holistic Works. (Febrero 2016). silybum-marianum (Cardo). Obtenido de: http://theholisticworks.com/2011/12/12/435/silybummarianum/ 